\title{
Modelling the Galaxy with orbital tori
}

\author{
James Binney \\ Rudolf Peierls Centre for Theoretical Physics, Keble Road, Oxford OX1 3NP, UK \\ email: binney@thphys.ox.ac.uk
}

Abstract. The principles and advantages of torus modelling are explained.

Keywords. Galaxy: dynamics, Galaxy: evolution

\section{What is torus modelling?}

Torus modelling (McMillan \& Binney 2008 and references therein) is a modification of Schwarzschild modelling in which orbits are numerically constructed as three-dimensional surfaces in six-dimensional phase space rather than as time sequences. These surfaces are topologically equivalent to 3-tori: if we identify each point of the floor of a room with the point of the ceiling that is vertically above it, and similarly identify corresponding points on the front and back walls, and on the right and left walls, the room becomes a 3 -torus. The "angle variables" $\theta_{1}, \theta_{2}, \theta_{3}$ are Cartesian coordinates for position in a room that has been thus made into a 3-torus: for example, as $\theta_{3}$ increments from 0 to $2 \pi$, the point moves vertically from floor to ceiling. Remarkably, as a star orbits through the Galaxy, its angle variables increase linearly in time, $\theta_{i}(t)=\theta_{i}(0)+\Omega_{i} t$, so its point moves in a straight line through its room-like orbital torus. Unless the frequencies $\Omega_{i}$ are rationally related $\left(\Omega_{1}: \Omega_{2}: \Omega_{3}=n_{1}: n_{2}: n_{3}\right.$ for integer $\left.n_{i}\right)$ the star eventually comes arbitrarily close to every point of its torus.

The natural labels for a torus are the three actions $J_{i}=(2 \pi)^{-1} \oint_{\gamma_{i}} \mathbf{v} \cdot \mathrm{d} \mathbf{x}$, where $\gamma_{i}$ is the path on which $\theta_{i}$ increments from 0 to $2 \pi$ with the other two angle variables held constant. Indeed the set of six coordinates $\left(J_{i}, \theta_{i}\right)$ are canonical coordinates for phase space. In particular the Poisson bracket of any two angle variables vanishes, $\left[\theta_{i}, \theta_{j}\right]=0$, so tori are null in the sense that the Poincaré invariant of any part of a torus vanishes $\left(\int \mathrm{d} \mathbf{v} \cdot \mathrm{d} \mathbf{x}=0\right)$.

The idea of torus-modelling is to use software that returns an orbit in terms of $\mathbf{x}(\boldsymbol{\theta})$ and $\mathbf{v}(\boldsymbol{\theta})$ rather than $\mathbf{x}(t)$ and $\mathbf{v}(t)$ as a Runge-Kutta integrator does. Since in this picture the orbit is identified with the torus, it is labelled by the actions $\mathbf{J}$. The following benefits flow from expressing orbits in this way.

- Given a point in space $\mathbf{x}$ we can readily find the values of $\boldsymbol{\theta}$ at which the star reaches that point and read off the velocities $\mathbf{v}(\boldsymbol{\theta})$ with which the star passes through that point. By contrast, if we are given $\mathbf{x}(t)$, we will in general search in vain for a time when the star is precisely at the given point and will have to settle for times when it is near. It will not be clear whether we have found times that give approximations to all the possible values of $\mathbf{v}$ at the given point.

- When we integrate orbits in time, the orbit is characterised by its initial conditions. The same orbit corresponds to infinitely many different initial conditions, so it is not clear how to do a systematic survey of phase space to obtain a representative sample of orbits. This difficulty does not arise in torus modelling because the actions are essentially unique labels of orbits and the orbits with actions in the range $\mathrm{d}^{3} \mathbf{J}$ occupy a volume $(2 \pi)^{3} \mathrm{~d}^{3} \mathbf{J}$ in 6-d phase space. 
- Actions are adiabatic invariants so we can relate orbits in different potentials: orbits with the same actions will deform into each other if we slowly deform the potential from that of one model to that of another. No such identification is possible if orbits are characterised by their initial conditions. On account of adiabatic invariance, the distribution function (DF) of a system such as a globular cluster is invariant as, for example, loss of gas modifies the cluster's density distribution and gravitational potential. Together with the previous item, adiabatic invariance make it straightforward to specify a galaxy model uniquely and to compare the orbital structures of models that have slightly differing potentials.

- Analytic formulae for $\mathbf{x}(\boldsymbol{\theta})$ and $\mathbf{v}(\boldsymbol{\theta})$ can be specified using much less data than are required to specify $\mathbf{x}(t)$ and $\mathbf{v}(t)$. Consequently tori greatly simplify manipulation of orbit libraries. Moreover, expressions for infinitely many tori can be obtained by interpolating between data for numerically obtained tori.

- There is a simple, intuitive connection between the functional form $f(\mathbf{J})$ of the DF and the real-space properties of the system it specifies (Binney \& Tremaine 2008 $\S 4.6)$. Moreover, given $f(\mathbf{J})$ there is a stable scheme for evaluating the self-consistent gravitational potential, which is not always the case when a DF of the form $f(E, \ldots)$ is specified.

- The orbit-averaged Fokker-Planck equation takes an exceptionally simple form in action space (Binney \& Tremaine 2008 §7.4.2), which should facilitate modelling of secular evolution.

- A set of orbital tori specify an integrable Hamiltonian which is very close to the true Hamiltonian. Perturbation theory works wonderfully well when this Hamiltonian is used as the point of reference (Kaasalainen 1994).

\section{How do we obtain tori?}

Analytic potentials (principally the potentials of the isochrone sphere and the multidimensional harmonic oscillator) provide analytic tori $\mathbf{x}(\boldsymbol{\theta})$, etc. We choose such a potential and refer to its structures as "toy" ones. A canonical transformation is used to map the toy torus with given actions $\mathbf{J}^{\prime}$ into the target phase space. The image torus is guaranteed to be null but in general it will not lie within a hypersurface on which the target Hamiltonian is constant, as an orbital torus must. We numerically adjust the coefficients that define the canonical transformation so as to minimise the rms variation $\Delta H$ in the target Hamiltonian over the image torus. Once $\Delta H$ is small enough, the target torus provides an excellent approximation to an orbital torus.

The canonical transformation $(\mathbf{J}, \boldsymbol{\theta}) \rightarrow\left(\mathbf{J}^{\prime}, \boldsymbol{\theta}^{\prime}\right)$ is specified by its generating function

$$
S\left(\mathbf{J}^{\prime}, \boldsymbol{\theta}\right)=\mathbf{J}^{\prime} \cdot \boldsymbol{\theta}+\sum_{\mathbf{n}} S_{\mathbf{n}}\left(\mathbf{J}^{\prime}\right) \mathrm{e}^{\mathrm{in} \cdot \boldsymbol{\theta}}
$$

where the sum is in principle over all vectors with integer components. The first term on the right generates the identity transformation, and the machine has to choose the coefficients $S_{\mathbf{n}}\left(\mathbf{J}^{\prime}\right)$ which are characteristic of the given orbit. Typically, a good approximation to an orbital torus can be obtained with a few tens of non-zero $S_{\mathbf{n}}$.

\section{References}

Binney, J. \& Tremaine, S., 2008, Galactic Dynamics, Princeton University Press, Princeton Kaasalainen, M., 1994, MNRAS, 268, 1041

McMillan, P. \& Binney, J., 2008, MNRAS, 390, 429 